This is a draft of a chapter that has been accepted for publication by Oxford University Press in the Oxford Handbook of Algorithmic Music, edited by Alex McLean and Roger Dean and published in 2018.

\title{
Chapter 11: Linking sonic aesthetics with mathematical theories
}

\author{
Andrew J. Milne \\ MARCS Institute, Western Sydney University, \\ Locked Bag 1797, Penrith, 2751, NSW, Australia
}

\begin{abstract}
Pure mathematics provides principles, procedures, and ways of thinking that can be fruitful starting points for music composition, performance, and algorithmic generation. In this chapter, a number of mathematical methods are suggested as useful ways to define and transform underlying musical structures such as meters and scales, and to realize these structures as finished pieces of music. The mathematical methods include the discrete Fourier transform, geometry, algebraic word theory, and tiling, and how these relate to musical features such as periodicity (or lack of periodicity), well-formedness, microtonality, canons, rhythmic hierarchies, and polyrhythms. The chapter closes with a detailed examination of a musical piece derived from the described processes.

Keywords: music, algorithmic music, math and music, perfect balance, wellformedness, Christoffel words, discrete Fourier transform, canons, Lindenmayer systems
\end{abstract}

Mathematics describes relationships between objects: collections of rules that determine how one or more object may be transformed into one or more other object, and the rich patterns that flow from these relationships. By a judicious mapping from mathematical structures to musical features, it becomes possible to imbue the latter with similarly rich structure and patterning - to link sonic aesthetics with mathematical theories.

In the following section, I will explore the application of mathematical techniques to mould the raw materials of music into interesting latent (as yet unrealized) structures. The focus will be on musical scales and meters, both periodic and non-periodic. In the section after that, I will explore some mathematically informed procedures that can produce musical realizations of these latent structures. These include musical canons, methods for generating self-similar and fractal-like forms, and the use of the Fourier transform to dynamically change pitch, timbre, and rhythm. At the risk of perpetuating the hegemonic "three-dimensional lattice" of discrete pitches, times, and timbres (Wishart 1983), most of the examples will use discrete events; despite that, many of the techniques described here are also applicable to smooth and dynamic changes of musical variables. In the third section, prior to the conclusion, I ground some of the abstractions by discussing a real-world musical realization. 


\section{Latent musical structures}

\section{Periodic}

Musical scales and meters are often thought of as periodic, which means they repeat at a regular pitch interval or time interval (non-periodic scales and meters are considered in a subsequent section). For example, the $\mathrm{C}$ major scale is assumed to repeat every octave, and ${ }_{4}^{4}$ time implies a repeating pattern of four beats - the first of which has greatest "weight". The most common periodicity in musical scales is at the octave. This likely arises from the (psycho-) acoustical similarity of harmonic complex tones an octave apart (the harmonics in the higher tone are all in the lower tone). Many common scales also have sub-periodicities that occur at subdivisions of the octave, such

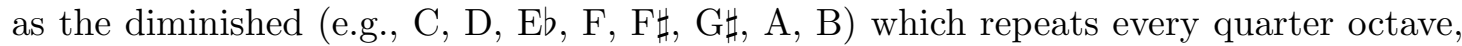
or the hexatonic (e.g., C, Db, E, F, G甘, A) which repeats every third-octave. Some scales do not exhibit periodicity at the octave: stretched octaves are commonly found in non-Western instruments (indeed intervals slightly larger than $2 / 1$ are typically heard, cross-culturally, as corresponding to an "octave" (Burns 1999)); repetition at the tritave (the 3/1 frequency ratio) has been proposed for instruments with only odd harmonics (Bohlen 1978; Mathews, Roberts, and Pierce 1984); Wendy Carlos (1987) developed alpha and beta scales (every step is 78 or 63.8 cents, respectively-neither of which produces a 1200 cents octave), and Gary Morrison (1993) has used an 88 cents equal tuning. Some examples of non-periodic structures are provided subsequently.

The periodicity of meters is exemplified by a straightforward Western meter that can be verbally counted as $1 \& 2 \& 3 \& 1 \& 2 \& 3 \& \ldots$, or by a more complex aksak meter (from the Balkan regions) such as $1 \& 2 \& \& 3 \& \& 4 \& 5 \& \& 1 \& 2 \& \&$ $3 \& \& 4 \& 5 \& \&$... (Fracile 2003). In distinction to scales, with meters there is no psychoacoustic imperative for any specific period size: there is not a specific interval of time that represents a "natural" period of equivalence; however, it is reasonable to assume that any such temporal period must be neither so short that it cannot be heard, nor so long that it cannot be remembered (perceptual and categorical time limits are discussed by London (2004)).

A periodic structure is naturally represented by a unit circle, which is represented in the complex plane by the formula $\mathrm{e}^{2 \pi \mathrm{i} x}$. (The complex plane is usually visualized as having real units increasing rightwards and complex units increasing upwards.) The letter i denotes the imaginary unit $\mathrm{i}=\sqrt{-1}$, which is orthogonal to the real unit 1 . A complex number is a linear combination of real and imaginary units, which means it can be thought of as a vector extending from the origin to some point in this two-dimensional space. The identity $\mathrm{i}^{2}=-1$ requires a special multiplication operation between such vectors, which is equivalent to summing their angles (measured anticlockwise from the ascending real line) and multiplying their length. When $x=0$, the $\mathrm{e}^{2 \pi \mathrm{i} x}$ formula corresponds to a vector extending from the circle's centre to a point at 3 o'clock; as $x$ increases, the vector's point moves anticlockwise around the circle; the circle is completed when $x=1$; hence, this function is periodic over the unit interval in $x$.

In much of this section, it will be useful to think of scales or meters with $K$ members as represented by a scale vector of $K$ complex numbers (i.e., as a vector of vectors). Each complex number in this vector represents a specific member of the scale or meter. Its phase -its anticlockwise angle with respect to the horizontal axis-represents the pitch class or time of that member. In the scale vector, the complex numbers are placed in phase order (i.e., they proceed anti-clockwise around the circle) - this latter requirement is necessary for some of the mathematics used later. For example, the 


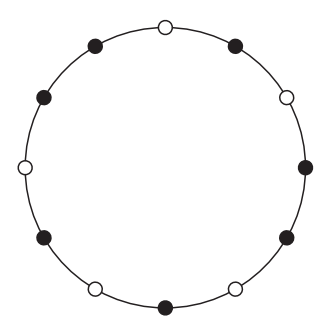

(a) Diatonic.

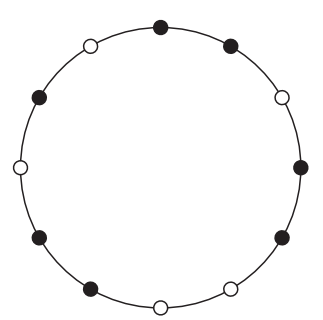

(b) Harmonic minor

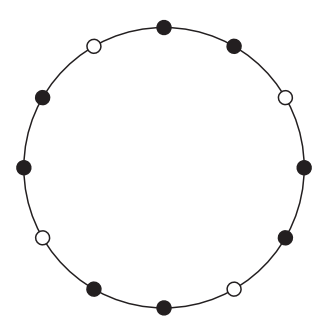

(c) Diminished.

Figure 1. Three scales represented by ordered points on the unit circle in the complex plane.

12-tone equal temperament (12-TET) diatonic major scale would have the scale vector $\boldsymbol{z}=\left(\mathrm{e}^{2 \pi \mathrm{i} 0 / 12}, \mathrm{e}^{2 \pi \mathrm{i} 2 / 12}, \mathrm{e}^{2 \pi \mathrm{i} 4 / 12}, \mathrm{e}^{2 \pi \mathrm{i} 5 / 12}, \mathrm{e}^{2 \pi \mathrm{i} 7 / 12}, \mathrm{e}^{2 \pi \mathrm{i} 9 / 12}, \mathrm{e}^{2 \pi \mathrm{i} 11 / 12}\right)$.

Figure 1 shows the diatonic major, harmonic minor, and diminished scales on the unit circle. The magnitude of each complex number can additionally be used to represent that member's "weight" (the magnitude of a complex number is changed by multiplying it by a real number). For example, the magnitude might represent loudness, or probability of occurring. In this case, some points will now lie outside the unit circle (greater weight), some will lie inside the circle (lesser weight); but, for the most part in this chapter, we will assume all members have unit weight. Under this representation, a circular shift (or rotation) represents transposition in pitch (for scales) or in time (for meters). Rotational symmetries (such as those in the diminished scale) represent subperiodicities. This method of representation allows for periodic structure to be mathematically analyzed in a number of ways, each of which provides useful information about its musical properties, and which may guide the generation of useful periodic patterns.

\section{Evenness and balance}

Two useful properties arise from the application of the discrete Fourier transform (DFT) to this circular characterization of periodic patterns in the complex plane. These are evenness and balance. Both are intuitively simple ideas that generalize aspects of many prevalent scales and meters, and can be used to guide the creation of unfamiliar scales and meters (including microtonal and non-isochronic).

A perfectly even rhythm or scale is one where every step size is identical. We are likely very familiar with such musical structures; for example, any regular (isochronous) beat is perfectly even, as is the common 12-TET tuning of the chromatic scale. Evenness quantifies the extent to which a pattern with $K$ elements approximates a perfectly even pattern also with $K$ elements. A perfectly balanced rhythm or scale is one where the mean position, or "centre of gravity", of all members around the circle is at the centre of the circle. Balance quantifies the proximity of the pattern's centre of gravity to the circle's centre.

Across the broad sweep of $K$-element patterns available in any given universe of $N$ equal steps, balance and evenness are typically highly correlated (e.g., approximately $.94)$; but there are notable examples where balance and evenness are quite different, as shown by the perfectly balanced patterns in Figure 2. Both balance and evenness seem plausibly important (archetypal) forms of organization, which may be relevant to musical structure. The importance of evenness in tonal and metrical structures has been extensively discussed (Rahn 1986; Clough and Douthett 1991; London 2004); prior to my own recent work, balance and its musical implications have not been explored in depth 
and it has often not been clearly disambiguated from evenness (Lewin 1959; Quinn 2004; Milne et al. 2015).

As mentioned above, the DFT of the scale vector provides a useful quantification of both properties. The $t$ th coefficient $(t=0,1, \ldots, K-1)$ of the DFT of the scale vector $\boldsymbol{z}$ (defined above) is given by

$$
\mathcal{F} \boldsymbol{z}[t]=\frac{1}{K} \sum_{k=0}^{K-1} \boldsymbol{z}[k] \mathrm{e}^{-2 \pi \mathrm{i} t k / K} .
$$

As shown by Amiot (2009a), the magnitude of the first coefficient (i.e., when $t=1$ ) gives the evenness of the pattern, which takes a value between zero and unity (the latter being perfect evenness):

$$
\begin{aligned}
\text { evenness } & =|\mathcal{F} \boldsymbol{z}[1]| \in[0,1], \text { where } \\
\mathcal{F} \boldsymbol{z}[1] & =\frac{1}{K} \sum_{k=0}^{K-1} \boldsymbol{z}[k] \mathrm{e}^{-2 \pi \mathrm{i} k / K}
\end{aligned}
$$

This equation can be broken down into three steps. Firstly, it calculates the circular displacements between each successive member of the scale vector and each successive member of a perfectly equal $K$-fold division of the period. Secondly, it calculates the circular variance of these values (circular variance has a maximal value of unity (Fisher 1993)). Finally, this circular variance is subtracted from unity. This means that if the displacements are all equal, their circular variance is zero and the pattern is perfectly even.

As shown by Milne et al. (2015), unity minus the magnitude of the zeroth coefficient (i.e., when $t=0$ ) gives the balance of the pattern, which takes a value between zero and unity (the latter being perfect balance):

$$
\begin{aligned}
\text { balance } & =1-|\mathcal{F} \boldsymbol{z}[0]| \in[0,1], \text { where } \\
\mathcal{F} \boldsymbol{z}[0] & =\frac{1}{K} \sum_{k=0}^{K-1} \boldsymbol{z}[k]
\end{aligned}
$$

This equation simply sums all the complex numbers (vectors) in the scale vector. If the result is a zero vector (all vectors have cancelled each other out), the pattern is perfectly balanced. For any given $N$ and $K$, there may be many different patterns with equivalent balance values (including perfect balance). Many perfectly balanced patterns have interesting and fairly complex structures, as illustrated in Figure 2 -in each of these patterns, the balance is achieved by combining regular polygons with coprime numbers of vertices. (Two or more numbers are coprime if their greatest common divisor is 1; e.g., 5 and 6 are coprime, but 4 and 6 are not because their largest common divisor is 2.) The scale in (a) is common in the Indian musical tradition (Bhairav that and Mayamalavagowla raga). In (b) and (c), some of the polygons have a weight of -1 , some have a weight of 1 , hence some of the vertices cancel out, thereby creating even more complex structures, which may provide interesting microtonal scales or non-isochronic beat rhythms. Perfectly balanced rhythms and scales such as these can be algorithmically generated by the XronoMorph application (Milne and Dean 2016; Milne et al. 2016). 


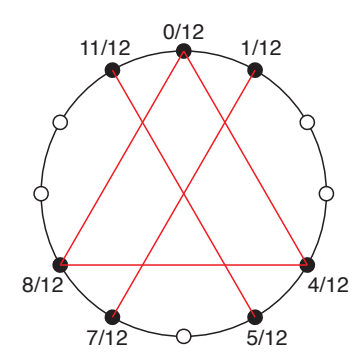

(a)

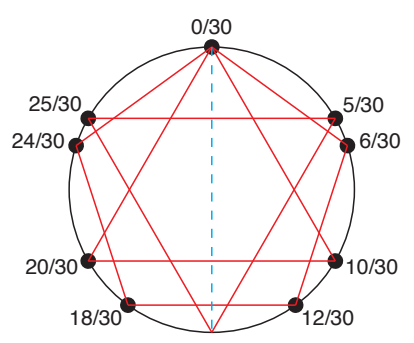

(b)

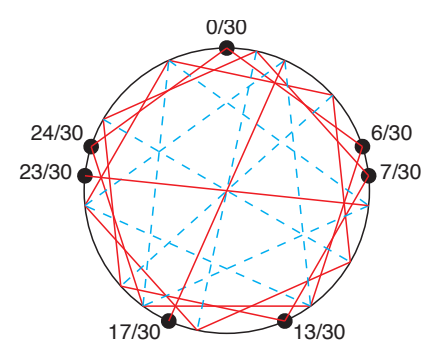

(c)

Figure 2. Three perfectly balanced patterns, all produced by combinations of regular polygons. The solid-line polygons have a weight of 1 , the dashed-line polygons have a weight of -1 . The sum of weights at each vertex is always 0 or 1 .

Perfect evenness, on the other hand, always represents a state of extreme simplicitysuch patterns have a distribution of intervals with the lowest possible entropy, and may be thought to have insufficient variation for musical purposes. An interesting solution is provided by maximizing evenness under an additional set of constraints. These are discussed in the following subsection.

\section{Well-formedness, Christoffel words, and hierarchies}

There is a useful way to constrain circular patterns (scales and meters) such that when evenness is maximized, the resulting pattern is not perfectly even, but also exhibits a remarkable set of structural properties that are hierarchical, complex, and interwoven. These are well-formed patterns. A well-formed pattern is one that has no more than two step sizes that are arranged so as to maximize the evenness of the resulting pattern (there are many alternative definitions, all of which lead to the same end result). Well-formed patterns were first described (in a musical context) by the microtonal theorist Erv Wilson (1975) (he denotes them moments of symmetry or MOS), and they were independently rediscovered and expressed in a different mathematical framework by Carey and Clampitt (1989).

Because the definition of well-formedness is somewhat abstract - it simply requires two step sizes, but there can be any number of either step, and each step can take any precise size - they are applicable to a wide variety of both familiar Western and non-Western scales (Carey and Clampitt 1989) and rhythms (Rahn 1986) as well as to experimental microtonal musical scales and rhythms (Carey 1998). Furthermore, such rhythms or scales may or may not be embedded within an equal temperament or isochronous pulse. Indeed, when the size ratio of the large interonset interval to the small takes on certain values based on the golden ratio $\phi=\frac{1+\sqrt{5}}{2} \approx 1.618$, and other so-called "metallic ratios" such as $\delta=\frac{2+\sqrt{8}}{2} \approx 2.414$ (silver ratio) and $\sigma=\frac{3+\sqrt{13}}{2} \approx 3.303$ (bronze ratio), the resulting rhythms are such that there is never any faster isochronous pulse that closely approximates the rhythm (Wilson 1997). Deeply non-isochronous rhythms such as these can be explored with XronoMorph.

A recent conceptualization of well-formed patterns is based on word theory (Clampitt, Domínguez, and Noll 2009; Domínguez, Clampitt, and Noll 2009). A word is an ordered list of letters taken from a finite alphabet. For example, we could denote the two step sizes by the letters $\ell$ (for "large") and $s$ (for "small"), and then any particular scale or meter is a different word made up from these letters; for example, the (well-formed) diatonic

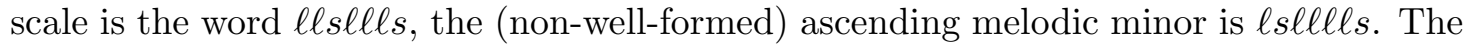
(non-well-formed) harmonic minor, for example, could not be represented by a word over 


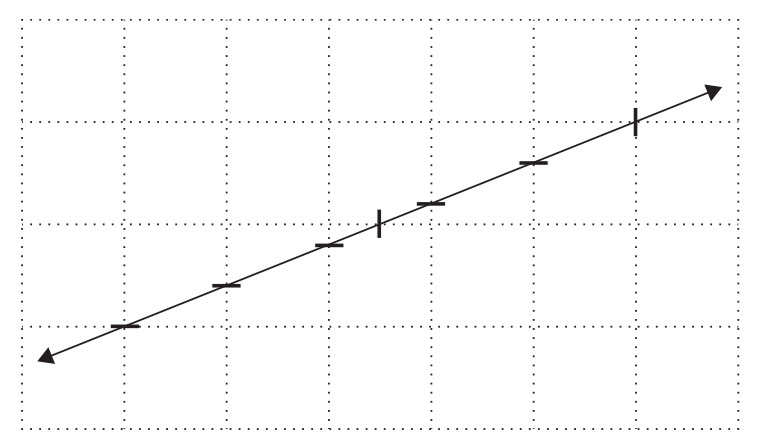

Figure 3. The cutting sequence of slope $2 / 5$. If the horizontal and vertical cuts represent $\ell$ and $s$, respectively, the well-formed diatonic word is produced (the Lydian mode, $\ell \ell \ell s \ell \ell s$ ). Note that the first and last cuts pass through corners, so the sequence of cuts is taken by moving the line infintesimally upwards. Because the slope is rational, the word is periodic.

a two letter alphabet because it has three step sizes. Well-formed words are special types of words known as Christoffel words or circular rotations, thereof (i.e., moving the last symbol to the start) (Clampitt, Domínguez, and Noll 2009).

For a Christoffel word that has a total of $j$ symbols of one type (e.g., $s$ ) and a total of $k$ symbols of the other type (e.g., $\ell$ ), the word is equivalent to the sequence of cuts a line of slope $j / k$ makes through horizontal and vertical lines of an integer grid (Berstel et al. 2009). This is illustrated in Figure 3.

When the sloping line cuts through a vertical line this represents a letter $\ell$, when it passes through a horizontal line this represents a letter $s$. When the line passes through a corner (as it does at the start and finish of the word), the first corner represents $\ell$ and the second represents $s$. This corresponds to shifting the sloping line vertically upwards an infinitesimal distance. (An alternative is for the first and second corners to represent $s$ and $\ell$, respectively, but the resulting word is simply a circular rotation of the other, (Lemma 2.7)). Clearly, the cutting sequence is periodic over the word of length $j+k$ because the same pattern repeats every time the line passes through a corner. Furthermore, the arrangement of the two letters is as even as possible.

A remarkable feature of well-formed patterns is that they form a rich hierarchy, whereby for any given well-formed pattern there is always another higher-level well-formed pattern that contains all the original's events and may contain more. For example, the pentatonic scale is embedded within the diatonic scale, which is embedded within the chromatic scale - all of these scales being well-formed. Because of the generality of the well-formed definition, these hierarchical relationships also hold for unfamiliar microtonal well-formed scales, and so generalize the notion of diatonic and chromatic. For example, Figure 4, shows the familiar Western hierarchy of well-formed scales, as well as two alternative microtonal well-formed scale (or metrical) hierarchies; there are numerous alternatives.

Hierarchies also apply to meters, where slower beats are typically accented more than intervening pulses; for example, in rock music, the slow rhythmic level may be enunciated by a psycho-acoustically prominent bass and snare, while a faster rhythmic level is enunciated by a more delicate cymbal. Well-formed hierarchies enable the creation of hierarchical meters in asymmetric time signatures (additive rhythms), as well as the deeply non-isochronous rhythms mentioned earlier.

Under the word theory representation, the generation of these higher-level rhythms can be easily understood (as discussed more extensively by Milne and Dean (2016)). For

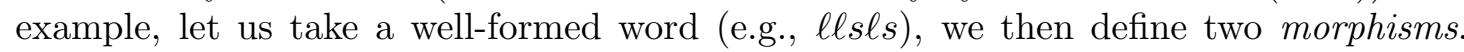
The first morphism performs the following two rewrites: $\ell \mapsto \ell s$ and $s \mapsto \ell$. The second morphism performs the following two rewrites: $\ell \mapsto \ell s$ and $s \mapsto s$. The first morphism 


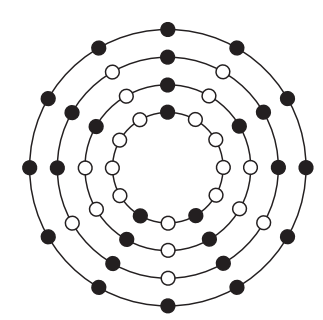

(a) 3 in 5 in 7 in 12 (the Western scalehierarchy).

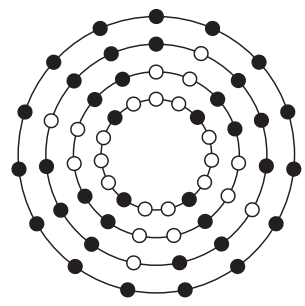

(b) 4 in 7 in 11 in 15 .

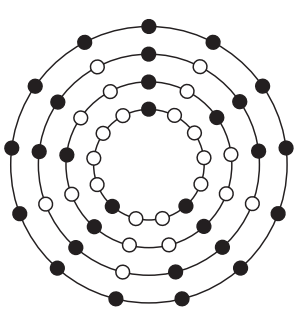

(c) 3 in 5 in 8 in 13 .

Figure 4. Three scale hierarchies: within each hierarchy, each successive level splits the large steps of the previous level.

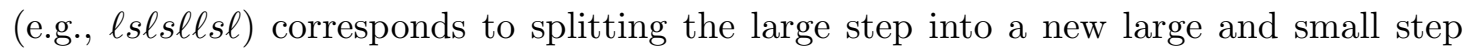
such that the old small step becomes the new large step, the second morphism (e.g., $\ell s \ell s s \ell s s)$ corresponds to splitting the large step into a new large and small step such that the old small step is the same as the new large step. In this way, each well-formed pattern can generate two direct descendants (one for each morphism), hence the number of different well-formed patterns quickly proliferates as we pass down the generations. (The above two morphisms can be equivalently characterized as L-systems, which are described later).

It is perhaps for all of these reasons that well-formed scales have been one of the most important organizational principles behind much recent work in microtonal music, as exemplified by members of microtonal internet fora and the xenharmonic wiki. In particular, there has been great interest in well-formed scales that contain numerous intervals that approximate just intonation intervals (rational frequency ratios, notably low integer ratios like $3 / 2,5 / 4,7 / 4$, etc., which are typically heard as consonant even when unfamiliar) (Erlich 2006; Milne, Sethares, and Plamondon 2008).

Such tunings of non-standard well-formed scales are interesting because they can connect familiar major and minor chords in unfamiliar ways; for example, certain chord progressions that, in standard Western tunings, start and end with precisely the same chord (e.g., I-vi-ii-V-I) will no longer do so and, importantly, vice versa. An example of the latter is TiHYL (Milne and Rolph 2014), which is in the scale system depicted in Figure 4(b) - the repeating eight-chord cycle in the introduction and middle section could be construed as $\mathrm{Bbm}-\mathrm{D} b-\mathrm{Dm}-\mathrm{E} 11-\mathrm{Fm}-\mathrm{A} b-\mathrm{Am}-\mathrm{B} 11-\mathrm{Bm}-\ldots$, but this final "Bm" chord is actually the same as the starting $\mathrm{Bbm}$. In this way, a musical cycle that is impossible in the standard diatonic/chromatic system has been realized. Another example utilizing the same scale hierarchy (but with a different tuning) is A Broken Stern (Milne 2012). A wide variety of well-formed scales with flexible tunings can be explored in the MIDI sequencer Hex (Prechtl et al. 2012) and the associated software at http://www.dynamictonality.com.

\section{Non-periodic}

Fibonacci sequences and Sturmian words

A Christoffel word is a finite-length word written in an alphabet of two letters, such that the two letters are as evenly distributed as possible. A Sturmian word is analogous except that it is non-periodic and of infinite length. Such words can, therefore, be used to generate well-ordered patterns which, remarkably, never repeat - they are a means to 


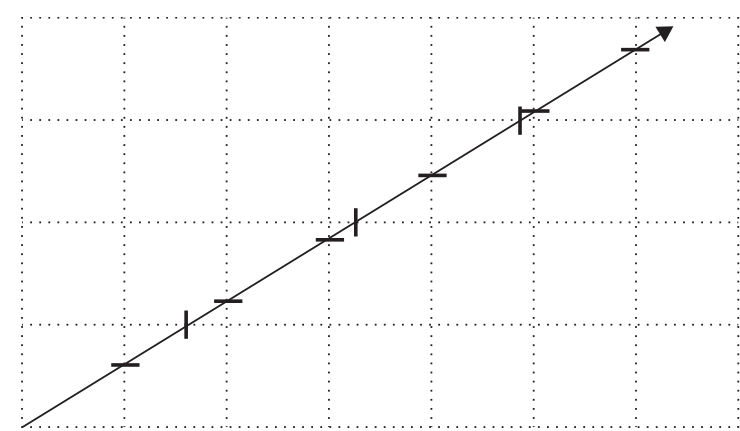

Figure 5. The cutting sequence of slope $1 / \phi=0.618 \ldots$ If the horizontal and vertical cuts are denoted 1 and 0 , respectively, the Fibonacci Sturmian word is produced. The first cut, which passes through the origin is typically ignored. Because the slope is irrational, the word is nonperiodic.

pattern the infinite. Sturmian words are the cutting sequence made through an integer grid by a line with a slope that is irrational (not a ratio of two integers). This is illustrated in Figure 5, which illustrates a line with the irrational slope of $1 / \phi$. When the sloping line passes through a horizontal grid-line this is denoted by a 1, when it passes through a vertical grid-line this is denoted by a 0 . The sequence of $1 \mathrm{~s}$ and $0 \mathrm{~s}$ is then read from left to right to make the Sturmian word. (The cutting sequence for any slope and its inverse are identical because they are just reflections about the line $y=x$; the slope $1 / \phi$ is used in Fig. 5 rather than $\phi$ because this means the figure can take a landscape orientation.) Because the line has irrational slope, it never passes through a vertex after the origin (the bottom-left corner) so the cutting sequence is non-periodic. If the slope had have been rational, the sequence of cuts would become periodic and would spell out a Christoffel (well-formed) word. In this way, we can see that Sturmian words are a generalization of Christoffel words.

These are the first 34 members of the sequences that arise from three well-known irrational numbers; each binary symbol might represent a different timbre or a time interval, and so forth (these are Sloane's integer sequences A005614, A144610, and A144609, respectively):

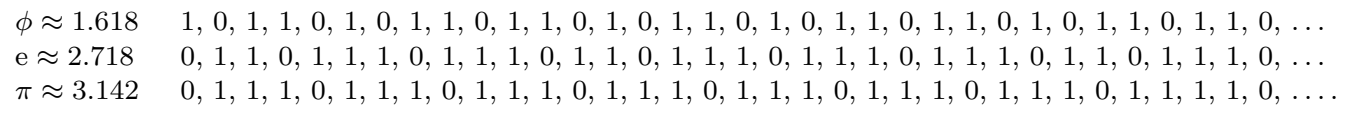

Because such sequences never repeat they may be used to generate infinitely-long meters or timbral shifts. Alternatively, arbitrarily sized segments of each such sequence can be extracted to form a finite structure. Sequences such as these, as generated by the golden section, have been musically utilized by Canright (1990, 2001) (e.g., Fibonacci Suite), and in numerous pieces by Mongoven (2013).

In distinction to the two-letter Fibonacci word described above, the more well-known Fibonacci sequence $(1,1,2,3,5,8,13,21, \ldots)$ has also been utilized to drive musical algorithms for pitch and time (White 1997). Over a longer musical time scale, Lendvai (1971) has claimed Bartok made use of Fibonacci sequences to structure his pieces (although these assertions are not without controversy (Howat 1983)).

A further generalization can be used to extend both the finite Christoffel words and the infinite Sturmian words. This is to allow for more than just two letters, but still ensure these letters are organized in an analogous fashion. This can be done by using cutting sequences through higher-dimensional grids - where the number of dimensions is the number of letters in the alphabet in which the words are written. Such words are known as billiard words because they replicate the pattern of bounces a perfect billiard 
ball would make when propelled inside a hypercube (Borel 2005; Bedaride and Hubert 2007).

\section{Other non-periodic structures}

An interesting method for structuring non-periodic scales are prime number scales (Dean 2009), which are based on multiplying a base frequency (not log-frequency) by the first 41, 51, 61, 71, or 91 successive primes. For instance, with a base frequency of $20 \mathrm{~Hz}$, the resulting scale would have frequencies (in $\mathrm{Hz}$ ) of $20 \times(2,3,5,7,11,13, \ldots)=$ $(40,60,100,140,220,260, \ldots)$. The resulting frequencies are, therefore, those of a fundamental and all of its prime numbered harmonics. The resulting scale has some frequency differences that occur more than once (e.g., there are three occurrences of a $40 \mathrm{~Hz}$ difference above) but the overall sequence is non-periodic because the primes are, themselves non-periodic. Furthermore, there will never be any two frequency ratios (log-frequencies) that occur more than once (this is a natural consequence of the fundamental theorem of arithmetic). The same procedure could be applied to event times as well as frequencies, thereby generating infinite-length rhythms which ascend through the primes.

Also worthy of note are the pitches used by Aboriginal Australians from the western desert area, which do not exhibit periodicity, but which do seem to contain repetitions of frequency differences rather than ratios (Will and Ellis 1996).

\section{Structured musical realizations}

Markov models, which assign probabilities to events and their transitions, and artificial intelligence systems (such as Petri nets), which are also based on transition rules, are commonly used to emulate established musical styles (Loy 2006; Nierhaus 2009). This can be achieved through statistical analyses of existing musical practice, and applying those insights to the probabilities and/or rules in the model. A more abstract approach is to make a natural mapping from structured latent materials to transition probabilities or rules. For instance, in a well-formed hierarchy, elements lower in the hierarchy (e.g., "diatonic" pitches or any microtonal generalization, thereof) may be assigned higher probabilities of occurring than elements higher in the hierarchy (e.g., "chromatic" pitches or any microtonal generalization, thereof). Advanced stochastic procedures, often operating on more abstract lines are detailed by Xenakis (1971) and are exemplified in his music (see also Dodge and Jerse (1997)).

Another approach is the use of complex dynamic systems. Such systems are capable of producing complex behaviour with simple interactions. For example, multi-agent systems that model flocking and swarming with simple rules can produce seemingly intelligent behaviour (think of a flock of birds where every bird suddenly changes to a new direction, apparently simultaneously). Indeed, swarming models have been used to mimic the interactions of freely improvising musicians (Blackwell and Bentley 2002; Blackwell and Young 2004; Unemi and Bisig 2004, 2005; Davis and Rebelo 2005). Other multi-agent systems have simulated evolutionary processes to generate musical structures (Miranda and Biles 2007).

In the following subsections, I consider processes that are more purely mathematical (i.e., less based on models of real-world phenomena). Such procedures may be used to create additional organization in keeping with, but also beyond, that implied by the latent materials. 


\section{Tone rows, tilings, and canons}

A musical realization where all elements of a set are played just once is a principled way of realizing a non-hierarchical latent structure (such as a scale/meter whose pitches/events are represented with a simple ordered list so that none of its members is privileged). It is principled in that it does not privilege - by repetition - any one element over any other.

To achieve this, one could simply make random selections, with a uniform distribution, across the set. But, typically, composers have sought greater organization. A classic method for achieving this is Schoenberg's tone row technique. A tone row is an ordered set of pitch classes, where no pitch class occurs more than once, and the tone row may be transformed, in toto, by any composition (succession) of transposition, inversion, and retrograde. If the pitch classes of the tone row are represented, in order, by a vector, these musical transformations are simple linear and invertible transforms (they can be performed by left-multiplying the tone row vector by a $12 \times 12$ matrix). Furthermore, each such row is played from start to finish. These rules ensure that, despite the large number of distinct transformations that can be made to the original tone row $(48$, in all), every possible member of the set of 12 pitch classes is played once and only once. It also ensures each tone row is related to every other (via transposition, inversion or retrograde), hence the rows exhibit symmetries with respect to their interval content. Related techniques can be applied to any musical dimension: indeed, the technique of total or integral serialism includes duration, loudness, timbre, articulation, and so forth (e.g., Babbitt, Nono, Boulez, Stockhausen, and other members of the Darmstadt School).

A generalization of the tone-row method is to use a partition of a universal set into disjoint (non-overlapping) subsets. The universal set is one that contains all elements of interest; for example, all twelve chromatic pitch classes, or all pulses in a meter. Then we iterate through the resulting subsets (this will be exemplified in the next paragraph). This ensures that all elements of the universal set are played once, but now with a structure resulting from the partition used. The patterning resulting from any given partition may be trivial but, as with evenness, when certain additional constraints are applied, interesting results occur.

One such constraint is that each subset is identical in form to every other: they differ only by their starting pitch or start time. This is exemplified by Figure 6: in a metrical context, the rhythm of each subset (all sequences $\mathrm{A}, \mathrm{A}, \mathrm{A}, \ldots$, or $\mathrm{B}, \mathrm{B}, \mathrm{B}, \ldots$, etc.) is denoted the inner rhythm (shown by the solid lines), the rhythm made by the repetitions (all sequences A, B, C, ...) is denoted the outer rhythm (shown by the dashed lines). Translating the inner rhythm by the outer rhythm, and summing them, results in every possible pulse being played once and only once. The inner and outer rhythms may or may not exhibit rotational symmetry (rotational symmetry means that there is a rotation by less than a full circle where the resulting pattern perfectly coincides with the unrotated version); for example, the inner rhythm in (a) has rotational symmetry because it is exactly the same whenever it is rotated by $1 / 2$ of a circle, the inner rhythm in (b) never exactly coincides when rotated by an angle less than the full circle and so does not have rotational symmetry, in both (a) and (b) the outer rhythm has rotational symmetry, in (c) it does not.

In a musical context, such rhythms mean that one voice might play a rhythmic pattern or melodic motif then, after an appropriate delay, a second voice plays exactly the same pattern then, after an appropriate delay a third voice plays exactly the same pattern, and so on. This procedure is, therefore, intimately related to the technique of musical canons, which have formed an important part of compositional procedure, notably in the works of Josquin des Prez, Obrecht, J. S. Bach, Haydn, and Olivier Messiaen: indeed, 


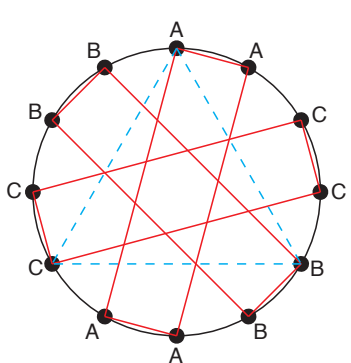

(a) Subperiodic inner and outer rhythms.

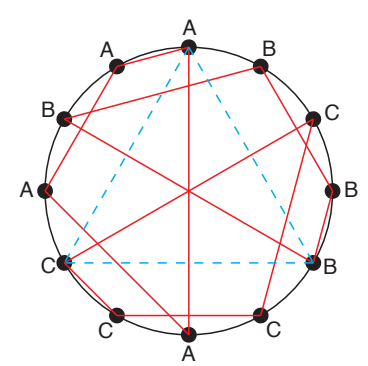

(b) Subperiodic outer rhythm

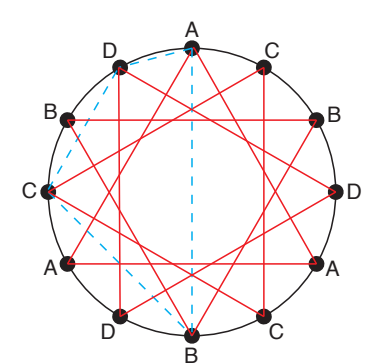

(c) Subperiodic inner rhythm.

Figure 6. Three regular complementary canons. The solid lines show the inner rhythms, the dashed lines show the outer rhythms. Note that (b) and (c) are complementary-the inner and outer rhythms have swapped. Any regular canon has such a complement.

regular tilings such as these were named regular complementary canons by Vuza in a series of papers (the most relevant to the present discussion being the third (Vuza 1992)) that first identified them and explored their mathematical properties.

Given a universal set with a specific number of equally spaced events, there will be only a limited number of such canons (trivially, only universal sets with non-prime numbers of elements can be tiled because the number of elements in each subset must be a divisor of the number of elements in the universal set). Hall and Klingsberg (2006, Thm. 6 with $r=n$ ) have shown that, for periods with $M$ equally spaced elements (e.g., regular pulses, or equal tempered pitches), inner rhythms with $n$ elements where $n$ is a divisor of $M$, and outer rhythms with rotational symmetry, the number of distinct regular complementary canons is

$$
\underset{\substack{M \\ \operatorname{gcd}(d, M / n)=1}}{\frac{1}{d, n}} \phi(d)(M / n)^{n / d}
$$

where gcd denotes the greatest common divisor, $\phi(d)$ is the Euler totient function which gives the number of positive integers no greater than $d$ that are coprime (defined earlier) with it, and $d \mid n$ are those $d$ which are factors of $n$. For example, a periodic meter with 24 isochronous pulses (e.g., two bars of ${ }_{8}^{12}$ ) has the following numbers of distinct regular complementary canons: 6 two-pulse patterns; 22 three-pulse patterns; 54 four-pulse patterns; 172 six-pulse patterns; 278 eight-pulse patterns; 172 twelve-pulse patterns. So clearly, there is still a wealth of different possibilities available. Interestingly, canons produced by non-periodic inner and outer rhythms form a special class known as Vuza canons, which are extremely rare and have been the subject of much investigation (Vuza 1992; Amiot 2009b).

Andreatta, Agon, and Amiot (2002) discuss other interesting canons where the subsets may be related not just by transposition but also by temporal retrogrades (or equivalently pitch inversion), and augmentations or diminutions. Canons such as these can be created and realized in the OpenMusic environment, which is designed for computer-aided music composition (Andreatta, Agon, and Chemillier 1999; Agon, Assayag, and Bresson 2006; Bresson, Agon, and Assayag 2011). 


\section{Self-similarities and fractals}

By definition, regular canons by translation (transposition), retrograde, and inversion exhibit self-similarity across those three different transformations. Patterns that exhibit self-similarity at all possible scalings ("zooms") are known as fractals. Fractals are highly organized, and their similarities to natural phenomena such as coastlines, mountain ranges, fungi, leaves, plants, trees, and so forth, is well documented (Mandelbrot 1983). From an algorithmic point of view, a useful aspect of fractals is that they can be generated by relatively simple formulae; furthermore, these algorithms typically generate sequences of numbers, which means each successive number can be naturally mapped to some parameter of each successive event (e.g., its pitch, duration, interonset interval, etc.).

\section{Fractal sequences}

Pressing (1988) details the musical implementation of a number of different functions, each of which produces a fractal sequence of numbers. His first example - the logistic map - is particularly illuminating and, given its complex results, is a remarkably simple algorithm. It was developed in the nineteenth century to model population dynamics, and generates successive values of $x$, indexed by $n$, with the following equation: $x_{n+1}=$ $a\left(x_{n}\left(1-x_{n}\right)\right)$, where $a$ is a parameter that controls the map's behaviour. When $3<a<4$, successive values of $x$ may oscillate between two, three, or more values but, at many $a$ in the range $3.57<a<4$, successive $x$ will oscillate chaotically between an infinite number of values. Pressing notes that $a$-values close to transitions between simple oscillations and chaos are particularly interesting because the resulting sequence shows unpredictability but also traces of cyclic behaviour. The stream of $x$ values can be straightforwardly mapped to any musical parameter of interest. For example, Pressing maps them to frequency by $2^{c x+d}$, where $c$ is the overall pitch range in octaves, and $d$ is the lowest frequency (the resulting frequencies were not quantized to 12-TET, so the melodies are microtonal). The above-mentioned pseudo-cyclic behaviour of the algorithm means that the resulting melodies also exhibit some degree of correlation over time-lags; in other words, they comprise "motifs" which approximately repeat.

In music, multiple musical variables (notably interonset interval, note duration, and pitch, for each of possibly multiple voices) must be controlled in tandem. Using different $a$-values for each variable means they lack any mutual influence, which Pressing describes as not musically desirable and resulting in too much unpredictability. In order to solve this issue, he tried fractal algorithms that generate higher-dimensional values: at each step, a pair, or more, of values is produced. These include maps using complex numbers, whose real and imaginary units naturally map into two dimensions, and quarternions, which have four independent components, and so could be used to control four musical variables at the same time. See http://www.australianmusiccentre.com.au/artist/ pressing-jeff for examples of Pressing's music.

\section{Lindenmayer systems}

Lindenmayer systems (also known as L-systems) were previously mentioned as a way to formalize the generation of successive levels in the hierarchy of well-formed patterns. These systems were introduced by Aristid Lindenmayer (1968) to model the development of simple multicellular organisms and were later used to model the forms created by fungi, plants and trees (Prusinkiewicz and Lindenmayer 1990). An L-system starts with a word (denoted an axiom) written in some alphabet. The word is then "rewritten" using a set 
of rules that determine exactly how each letter is to be transformed. In this way, an Lsystem is closely related to a generative grammar (Chomsky 1963): the difference is that, in a grammar, the rewriting rule is not necessarily applied to every letter in the word; in an L-system, the rewriting is always applied to all letters (applications of generative grammars to music are not covered here; see Jones (1981) and Rohrmeier (2011)). The rewriting process can be repeated indefinitely by applying the same rewriting rules to the result of the previous rewrite. This results in words exhibiting fractal self-similarities.

To give these symbolic words aesthetic value, they must be mapped to musical variables. Also, it is necessary to define an order in which a word's symbols are "read". Typically, they are simply read from left to right (or right to left). A common approach is to produce a visual representation of the word using turtle drawing (Papert 1980), exemplified below, then map features of the resulting graphic to musical variables. For example, there may be an alphabet with three symbols $F$, + , and - , where $F$ means draw a line of unit length, + means turn clockwise by $90^{\circ},-$ means turn anticlockwise by $90^{\circ}$. Using this drawing scheme with the axiom $F+F+F+F$ and three rewriting rules: (1) $F \mapsto F+F-F-F F+F+F-F$; (2) $+\mapsto+$; (3) $-\mapsto-$ results in the quadratic Koch curve - a highly patterned non-intersecting fractal comprising horizontal and vertical segments (Prusinkiewicz 1986). Prusinkiewicz suggests moving through the word from left to right and using the height of each line segment as its pitch height (alternatively, this could be scale degree), and its horizontal length as its duration or interonset interval - as if each horizontal line segment is a MIDI event in a software sequencer or piano roll.

Nelson (1996) extends this idea by using different turning angles $\left(101^{\circ}\right.$ or $107^{\circ}$ rather than $90^{\circ}$ ). The resulting graphic was then further manipulated with nonlinear stretches, twists and warps. The height of each successive vertex (the points at which the line changes direction) was used to specify each successive pitch, and the horizontal distance between successive vertices specified the respective note's duration.

To deal with the previously mentioned problem of ensuring interrelationships between multiple simultaneous voices, Prusinkiewicz (1986) suggests the use of L-system techniques to model the branching found in, for example, trees where a main trunk will have branches, which themselves have sub-branches (twigs), and so on. To achieve this, additional branching symbols [ and ] are added to the alphabet: the first marks the start of a branch, the second marks the end of the branch and instructs the drawing turtle to return to the position when [ was first encountered. The use of such techniques to generate distinct musical lines has been explored by DuBois (2003), and led to his development of the [jit.linden] object in Max (https://cycling74.com). Kevin Jones (1989) discusses a number of interesting musical applications of L-systems, including multidimensional generalizations, which naturally lend themselves to multidimensional musical spaces.

The process of choosing useful L-system rewriting rules and axioms, as well as mapping the resulting symbols to musical variables, is far from trivial. DuBois (2003) suggests using statistical analyses of the symbols in the resulting word to ensure the upwards and downwards movements in pitch, duration, loudness, and so on, are reasonably balanced (e.g., if a word uses $U$ to represent a specific ascending pitch interval, and $D$ to represent a specific descending pitch interval, and say a statistical analysis of the resulting word shows there are twice as many $U$ symbols as there are $D$ symbols, it might be sensible to make the ascending interval half the size of the descending so as to avoid a general upwards drift in pitch). Soddell and Soddell (2005) take a different approach in that they chose a specific biological form - the fungus Geotrichum - and use the stochastic L-system that best models its growth. 
Random noise exhibits scaling similarity in that the general appearance of a noise waveform does not change as you zoom the time dimension; put differently, you can slow down or speed up a recording of noise and it will sound the same (assuming an adequate recording and playback system). The continuum of noise is often categorized into three types, each of which has a different high-frequency roll-off: white noise has constant power spectral density across frequency; pink noise has a power spectrum inversely proportional to frequency $(1 / f)$; brown noise (named after the related brownian motion, which describes the random movements of particles in a fluid) has a power spectrum inversely proportional to squared frequency $\left(1 / f^{2}\right)$. In addition to their scaling self-similarities, of particular importance with respect to their musical utility are their self-similarities across time. A simple way to measure self-similarity with respect to time is to perform autocorrelation on the signal. In general, white noise has zero autocorrelation (other than at lag zero), so exhibits no similarities across time lags. Interestingly, any noise with power spectral density roll-off exhibits correlations across time-lags: brown noise has strong correlations over small time scales, which gradually decreases as the time lag increases; pink noise has moderate correlation across all time scales, even across large time scales.

By mapping successive noise values to musical variables of successive events, noise can be used to generate stochastic realizations that also exhibit self-similarity across scale (augmentation/diminution), time or pitch. For this purpose, Voss and Clarke (1978) suggested that white noise is too random, brown is too correlated, but pink is just right; they also presented empirical evidence that pink noise is prevalent in music. Nettheim (1992), however, subsequently demonstrated that, under a more precise analytical method, the pitch distributions in common-practice music (for lags of up to four bars) tend more towards $1 / f^{2}$ than $1 / f$. Recent analyses of John Coltrane's solos also show spectra closer to $1 / f^{2}$ than to $1 / f$ (Charyton 2015). Regardless of its precise specifications, if a stochastic process is required, then using noise with a downwards spectral slope is a means to produce similarities across both scaling and translation. Jones (1989) demonstrates an interesting application of brown noise to "walk" the frequencies of sine waves across different harmonics of the same fundamental frequency, thereby producing a continuously evolving timbral texture.

\section{Fourier scratching}

To end, let us return to the first-used mathematical formalization: the representation of a periodic pattern as a scale-vector in the complex domain, and the discrete Fourier transform of this vector to produce a DFT-vector that quantifies balance and evenness. The Fourier scratching technique, developed by Amiot, Noll, Andreatta, Agon, and Carlé (Amiot et al. 2006; Carlé and Noll 2010; Milne et al. 2011) reverses this process, and treats the DFT-vector as a set of user-adjustable parameters that generate - with an inverse DFT - a scale- or meter-like IDFT-vector. The coefficients of this generated pattern are cycled through, in order, and used to trigger pitches or timbres in a circular space.

More concretely, imagine we have a circular keyboard with $K$ keys each of which occupies a circular sector (wedge), and each different sector may subtend a different angle (the keys may have differing widths). For example, a well-formed scale with four large steps and three small could be represented by four wide sectors and three narrow arranged in an appropriate circular order. Now imagine a virtual robot with $N$ "fingers", 
Table 1. The five rhythmic levels in the well-formed hierarchy used in Babylon 19|30.

\begin{tabular}{|c|c|c|c|c|c|}
\hline level & morphism & pattern & signature & $\operatorname{size}(\ell)$ & $\operatorname{size}(s)$ \\
\hline 1 & & $\ell s \ell$ & $2 \ell, 1 s$ & 8 & 3 \\
\hline 2 & $\ell \mapsto \ell s, \quad s \mapsto s$ & $\ell s s \ell s$ & $2 \ell, 3 s$ & 5 & 3 \\
\hline 3 & $\ell \mapsto \ell s, \quad s \mapsto \ell$ & 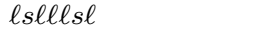 & $5 \ell, 2 s$ & 3 & 2 \\
\hline 4 & $\ell \mapsto \ell s, \quad s \mapsto \ell$ & 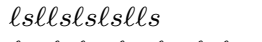 & $7 \ell, 5 s$ & 2 & 1 \\
\hline 5 & $\ell \mapsto \ell s, \quad s \mapsto s$ & 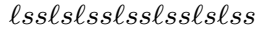 & $7 \ell, 12 s$ & 1 & 1 \\
\hline
\end{tabular}

such that the first finger is placed at the phase of the IDFT-vector's zeroth coefficient, the second finger is placed at the phase of the first coefficient, and so on until the positions of all $N$ fingers are defined. A regular pulse is used to trigger finger 1, then finger 2, up to finger $N$, then back to finger 1 , and so on. Given a circular keyboard of $K$ keys, the locations of the $N$ fingers around the circle will determine which of the $K$ notes they play, and when. Furthermore, the magnitude of each coefficient determines the velocity of that finger's virtual strike. Note that smooth variations of the parameters in the DFT-vector will smoothly vary the positions and velocities of the robot's fingers, and that the robot's fingers can cross thereby changing the order in which keys are played. In this way, complex patterns of pitches and loudnesses can be quickly created and smoothly morphed between. This example has used the metaphor of a standard pitch-based keyboard, but the coefficients' phases can also be applied to any musical variable that can be made periodic (e.g, a loop "drawn" through a multidimensional parameterization of a timbral/spectral space). In this case, the circular keyboard may be entirely dispensed with so the robot fingers directly trigger specific points within the continuum.

\section{A musical realization}

In this section, I describe an actual musical realization of some of the previously discussed ideas. As mentioned in Sec. 1.1.2, I have previosuly used well-formedness to structure microtonal scales; in the piece I discuss here, I instead apply well-formedness to musical rhythms. I additionally apply, for the first time, the principle of perfect balance.

Babylon 19|30 alternates between two multi-layered rhythmical structures. The first is a well-formed structure in a time signature of $\frac{19}{8}$, the second is perfectly balanced rhythm in ${ }_{8}^{30}$ (or, for comprehensibility, ${ }_{8}^{15}$ ). The harmonic contents of the loops vary but are kept relatively simple (essentially diatonic with soft "modal" dissonances) so as not to distract from the rich and complex rhythmical structure. The rhythms were generated as live MIDI by XronoMorph and sent to synthesizers. Additional physical instrument parts were then overdubbed.

The well-formed section has five rhythmic levels, where each successive level is derived by splitting the long durations of the next lower level. The lowest level comprises just three beats, two of which are long ( 8 pulses), one of which is short ( 3 pulses). The long beats are split to make the next higher level rhythm, and this is done successively until the fifth and final level of 19 equally-sized beats is formed. Each of the levels is characterized in Table 1. Interestingly, this hierarchy corresponds precisely to that found in the pentatonic-diatonic-chromatic scale hierarchy when a 19-TET tuning is used (I did not plan this in advance, and only noticed it while writing up this description).

A result of the well-formed method of generating successive levels is that every rhythmic event is duplicated in all higher-levels. For example, all three beats in the first level are additionally played by the remaining four levels. Naturally, this gives a strong accent to 


Table 2. The five complementary well-formed rhythmic levels
actually used in Babylon $19 \mid 30$. The pitches assigned to each
level transition every few repetitions to give harmonic move-
ment and melodic variety. The offset is the number of pulses
after the start of the period that that level's first beat plays.
\begin{tabular}{llllll} 
level & offset & pattern & signature & $\operatorname{size}(\ell)$ & $\operatorname{size}(s)$ \\
\hline 1 & $0 / 19$ & $\ell s \ell$ & $2 \ell, 1 s$ & 8 & 3 \\
2 & $5 / 19$ & $\ell s$ & $1 \ell, 1 s$ & 11 & 8 \\
3 & $3 / 19$ & $\ell s$ & $1 \ell, 1 s$ & 11 & 8 \\
4 & $2 / 19$ & $\ell s s \ell s$ & $2 \ell, 3 s$ & 5 & 3 \\
5 & $1 / 19$ & $\ell s \ell \ell \ell s$ & $5 \ell, 2 s$ & 3 & 2 \\
\hline
\end{tabular}

Table 3. The six simultaneously playing balanced components in the second section of Babylon 19|30. The offset is the number of pulses after the start of the period that that component's first beat plays.

\begin{tabular}{lll}
\hline balanced polygon & offset & pitch \\
\hline regular pentadecagon & $0 / 30$ & C7 \\
regular decagon & $2 / 30$ & G5 \\
regular hexagon & $4 / 30$ & G4 \\
irregular heptagon & $0 / 30$ & D3 \\
regular triangle & $2 / 30$ & F2 \\
regular pentagon & $3 / 30$ & A1 \\
\hline
\end{tabular}

low-level beats, and amplifies the inherently hierarchical nature of well-formed rhythmic structures. However, there is an interesting alternative strategy, which is to treat each successive level as the complement of all lower levels, so it plays only when no lower level is also playing. For example, say we have a lower level which, if expressed as a scale rather than as a rhythm, corresponds to the white-note diatonic scale (as in level 3 of Table 1), while the next higher level corresponds to a twelve-pitch chromatic scale (as in level 4 of Table 1). Then, instead of playing all twelve events in the latter rhythm, only those events not occurring in the lower-level pattern are played. Using the scalic analogy, this means using only the black-note pentatonic scale, which is the complement of the diatonic in a chromatic universe. Interestingly, these complementary well-formed rhythms are themselves well-formed (Amiot 2007, Proposition 3.2), but they are displaced with respect to each other, so they never coincide. The resulting patters are shown in Table 2. This creates a sparser rhythmic structure that is somewhat reminiscent of the multiple interlocking parts used by latin or gamelan percussion ensembles - although each individual part is relatively simple, in combination, they produce a complex and interwoven totality.

The perfectly balanced section of the piece comprises six independent rhythmic components (I use the word component rather than level because, unlike well-formed rhythmic structures, there is no implied hierarchy in perfectly balanced rhythmic structures). These are detailed in Table 3, where they are ordered by their pitch height. The irregular heptagon is that shown in Figure 2(c). All the other polygons are regular, and have numbers of vertices $(3,5,6,10$, and 15) that factor into 30 , which is the number of underlying pulses in the period.

Both types of rhythmic pattern are illustrated as polygons in Figure 7 and staff notation, with pitches, in Figure 8. An important aspect of the algorithmic process used for this piece is that, without further user-intervention, every vertex of any given polygon (both well-formed and balanced) produces the same pitch value. This introduces a rewarding compositional constraint to work with. Interestingly, it also means that when 


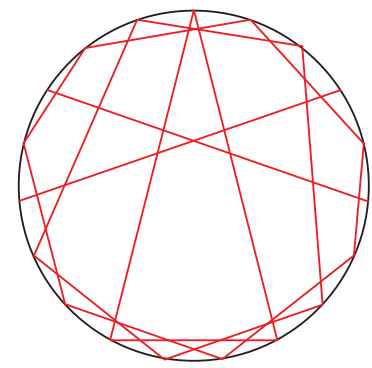

(a) Well-formed.

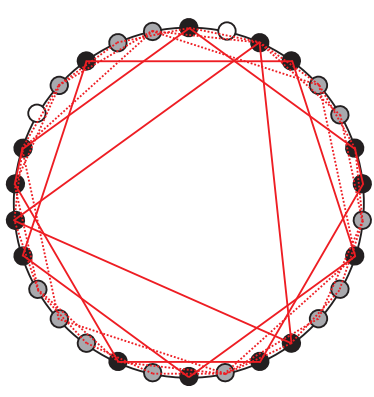

(b) Perfectly balanced.

Figure 7. The two rhythmical patterns in Babylon 19|30 visualized as polygons inscribed in a periodic circle. Imagine a playhead rotating clockwise around the circle, and outputting a MIDI note whenever it encounters a polygon vertex. The pitch, velocity, and duration of that MIDI note is a determined by the polygon to which the vertex belongs (the user inputs these values for each polygon). In the well-formed rhythm, precisely one event occurs at every nineteenth (pulse) division of the period. In the balanced rhythm, the three lowest-pitched polygons delineate a bass pattern (solid lines and dark disks), while the others have a more percussive or melodic effect (dotted lines and grey disks). At two out of the thirty (pulse) divisions of the period, no event occurs (white disks). In the piece, I have made the lengths of the periods of the well-formed and balanced rhythms take the ratio 19:30 to equalize their pulse tempos.

the pitch values and vertices of two polygons are close, they are likely to be perceptually streamed into a single melodic line. As the music progresses, I have successively applied different pitch values to different polygons, which results in harmonic shifts as well a variety of implied or "half-heard" melodies emerging from the whole.

XronoMorph greatly facilitates the production of rhythms such as these. It is unlikely I would have been able to produce a piece of music like this without it; in this sense, the algorithms and the mathematical structures it embodies have expanded my creative facility. Furthermore, rhythms such as these - despite their accessible sound - can be extremely difficult to perform. In other recent live work, I have used the same algorithmic routines to generate well-formed beats on-the-fly with both abrupt and smoothly changing structures. Music such as this is genuinely impossible to achieve without computational means.

Using the algorithmic approach described here, intelligent compositional input is still required - not all well-formed and perfectly balanced rhythms, or transitions between them, will sound appropriate. Furthermore, effective choices for pitches and durations are still required. These latter choices could feasibly be done algorithmically too-in earlier experiments we have used well-formed rhythms to drive Dean's Serial Collaborator (Dean 2013) to produce rhythmically informed serial transformations of previously written tone rows.

\section{Conclusion}

As mentioned in the introduction, although the procedures described in this chapter have been mostly exemplified by discrete pitches and times, many of them are also applicable to sonic gestures in the continuum of musical space. For example, well-formedness, fractal structures, and (despite its name) the discrete Fourier Transform, lend themselves to smooth and dynamic changes of continua of time, space, timbre, and so forth. I hope that the distinction between latent musical structures and processes by which these are realized has usefully reflected the way that compositional processes typically unfold - in 

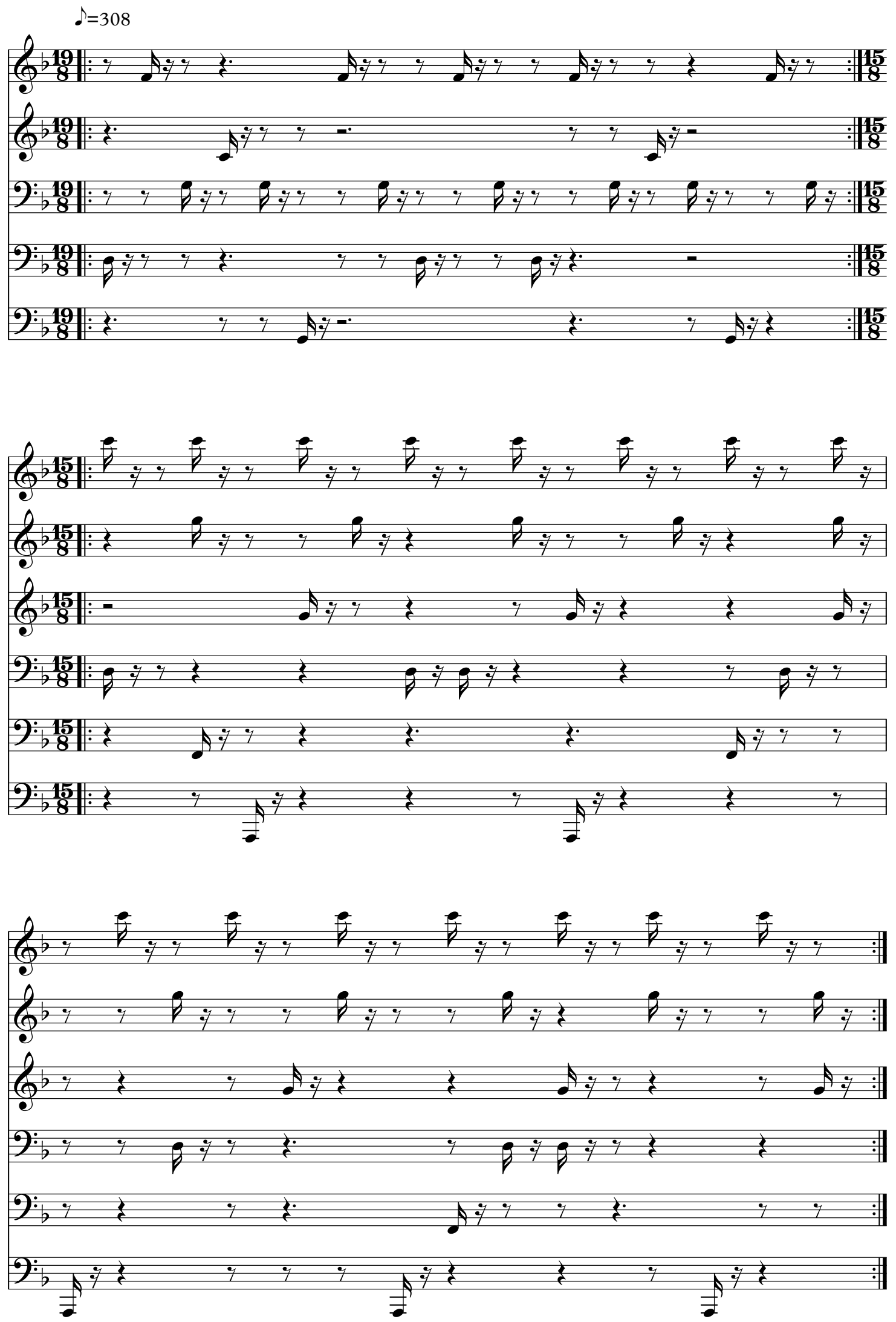

Figure 8. Two loops from Babylon 19|30 in staff notation. The one-measure loop in 19-time is well-formed. The two-measure loop notated in 15-time is perfectly balanced. In both loops, each polygon is notated with a separate staff so as not to specify where melodic streaming may arise across the levels (hocketing). For readability, all staffs are vertically arranged in order of pitch height, which means the well-formed staffs are not arranged as in Table 2. 
both the human and the computational context. We choose our palette, we paint our picture.

\section{References}

Agon, C., G. Assayag, and J. Bresson. 2006. The OM Composer's Book. Vol. 1. Editions Delatour France/Ircam.

Amiot, E. 2007. "David Lewin and maximally even sets." Journal of Mathematics and Music 1 (3): 15772 .

2009a. "Discrete Fourier transform and Bach's good temperament." Music Theory Online 15 (2). 2009b. "New perspectives on rhythmic canons and the spectral conjecture." Journal of Mathematics and Music 3 (2): 71-84.

Amiot, E., T. Noll, M. Andreatta, and C. Agon. 2006. "Fourier oracles for computer-aided improvisation." In Proceedings of the International Computer Music Conference, 99-103. New Orleans, LA, US.

Andreatta, M., C. Agon, and E. Amiot. 2002. "Tiling problems in music composition: Theory and implementation." In Proceedings of the International Computer Music Conference, 156-63. Göteborg, Sweden.

Andreatta, M., C. Agon, and M. Chemillier. 1999. "OpenMusic et le problème de la construction des canons musicaux rythmiques." In Actes des sixièmes Journées d'Informatique Musicale, 179-85. Paris.

Bedaride, N., and P. Hubert. 2007. "Billiard complexity in the hypercube." Annales de l'institut Fourier 57 (3): 719-38.

Berstel, J., A. Lauve, C. Reutenauer, and F. V. Saliola. 2009. Combinatorics on Words: Christoffel Words and Repetitions in Words. American Mathematical Society.

Blackwell, T., and P. Bentley. 2002. "Improvised music with swarms." In Proceedings of the 2002 Congress on Evolutionary Computation, 2002. 2:1462-67. IEEE.

Blackwell, T., and M. Young. 2004. "Self-organised music." Organised Sound 9 (2): 123-36.

Bohlen, H. 1978. "13 Tonstufen in der Duodezime." Acustica 39 (2): 76-86. http://www.huygensfokker.org/bpsite/publication0178.html.

Borel, J.-P. 2005. "A geometrical approach to palindromic factors of standard billiard words." Discrete Mathematics and Theoretical Computer Science 9 (2): 195-212.

Bresson, J., C. Agon, and G. Assayag. 2011. "OpenMusic: Visual programming environment for music composition, analysis and research." In Proceedings of the 19th ACM international conference on Multimedia. Scotsdale, AZ, USA.

Burns, E. M. 1999. "Intervals, scales, and tuning." In The Psychology of Music, 2nd, edited by D. Deutsch, 215-64. New York: Academic Press.

Canright, D. 1990. "Fibonacci gamelan rhythms." Journal of the Just Intonation Network 6 (4): 4.

. 2001. "Fibonacci Suite for retuned piano, seven hands." Accessed 2001. http://home.comcast. net/ dcanright/suite/index.htm.

Carey, N. 1998. "Distribution modulo one and musical scales." PhD diss., University of Rochester.

Carey, N., and D. Clampitt. 1989. "Aspects of well-formed scales." Music Theory Spectrum 11 (2): 187206.

Carlé, M., and T. Noll. 2010. "Fourier-scratching." In $\{$ SOUNDINGCODE $\}$ SuperCollider Symposium 2010. Berlin.

Carlos, W. 1987. "Tuning: At the crossroads." Computer Music Journal 11 (1): 29-43.

Charyton, C. 2015. "The impact of improvisation on creativity: A fractal approach." Chap. 8 in Creativity and Innovation Among Science and Art: A Discussion of the Two Cultures, edited by C. Charyton, 153-78. Springer.

Chomsky, N. 1963. "Formal properties of grammars." In Handbook of Mathematical Psychology, edited by R. Luce, R. Bush, and E. Galanter, vol. II. New York: Wiley.

Clampitt, D., M. Domínguez, and T. Noll. 2009. "Plain and twisted adjoints of well-formed words." In MCM 2009, edited by E. Chew, A. Childs, and C.-H. Chuan, 65-80. CCIS. Berlin Heidelberg: Springer-Verlag.

Clough, J., and J. Douthett. 1991. "Maximally even sets." Journal of Music Theory 35 (1/2): 93-173.

Davis, T., and P. Rebelo. 2005. "Hearing emergence: Towards sound based self organisation." In Proceedings of the International Computer Music Conference. Barcelona, Spain.

Dean, R. T. 2009. "Widening unequal tempered microtonal pitch space for metaphoric and cognitive purposes with new prime number scales." Leonardo 42 (1): 94-95. 
2013. "The Serial Collaborator: A meta-pianist for real-time tonal and non-tonal music generation." Leonardo 47 (3): 260-61.

Dodge, C., and T. A. Jerse. 1997. Computer Music: Synthesis, Composition, and Performance. Second. Schirmer.

Domínguez, M., D. Clampitt, and T. Noll. 2009. "WF scales, ME sets, and Christoffel words." In Mathematics and Computation in Music - MCM 2007, edited by T. Klouche and T. Noll, 37:477-88. CCIS. Berlin Heidelberg: Springer-Verlag.

DuBois, R. L. 2003. "Applications of Generative String-Substitution Systems in Computer Music." PhD diss., Columbia University.

Erlich, P. 2006. "A middle path between just intonation and the equal temperaments, part 1." Xenharmonikôn 18:159-99.

Fisher, N. I. 1993. Statistical Analysis of Circular Data. Cambridge University Press.

Fracile, N. 2003. "The aksak rhythm, a distinctive feature of the Balkan folklore." Studia Musicologica Academiae Scientiarum Hungaricae 44 (1): 191-204.

Hall, R. W., and P. Klingsberg. 2006. "Asymmetric rhythms and tiling canons." The American Mathematical Monthly 113 (10): 887-96.

Howat, R. 1983. "Bartók, Lendvai and the principles of proportional analysis." Music Analysis 2 (1): 69-95.

Jones, K. 1981. "Applications of Stochastic Processes." Computer Music Journal 5 (2): 45-61.

- 1989. "Generative models in computer-assisted musical composition." Contemporary Music Review 3:177-96.

Lendvai, E. 1971. Béla Bartók: An analysis of his music. London: Kahn \& Averill.

Lewin, D. 1959. "Re: Intervallic Relations between two collections of notes." Journal of Music Theory 3 (2): 298-301.

Lindenmayer, A. 1968. "Mathematical models for cellular interaction in developmen, parts I and II." Journal of Theoretical Biology 18:280-315.

London, J. 2004. Hearing in Time: Psychological Aspects of Musical Meter. Oxford University Press.

Loy, G. 2006. Musimathics. Vol. 1. MIT Press.

Mandelbrot, B. B. 1983. The Fractal Geometry of Nature. New York: W. H. Freeman.

Mathews, M. V., L. A. Roberts, and J. R. Pierce. 1984. "Four new scales based on nonsuccessive-integerratio chords." Journal of the Acoustical Society of America 75 (S1): S10.

Milne, A. J. 2012. A Broken Stern. The Stern Brocot Band. (V. Angelis, S. Holland, A. J. Milne). SoundCloud, https://soundcloud.com/andrew-j-milne/a-broken-stern-2012.

Milne, A. J., D. Bulger, S. Herff, and W. A. Sethares. 2015. "Perfect balance: A novel organizational principle for musical scales and meters." In Mathematics and Computation in Music: Proceedings of the 5th International Conference, edited by T. Collins, D. Meredith, and A. Volk, 9110:97-108. LNCS. Berlin: Springer.

Milne, A. J., M. Carlé, W. A. Sethares, T. Noll, and S. Holland. 2011. "Scratching the Scale Labyrinth." In Mathematics and Computation in Music: Proceedings of the 3rd International Conference, edited by C. Agon, E. Amiot, M. Andreatta, G. Assayag, J. Bresson, and J. Mandereau, 6726:180-95. LNCS. Berlin: Springer.

Milne, A. J., and R. T. Dean. 2016. "Computational creation and morphing of multilevel rhythms by control of evenness." Computer Music Journal 40 (1): 35-53.

Milne, A. J., S. A. Herff, D. Bulger, W. A. Sethares, and R. Dean. 2016. "XronoMorprh: Algorithmic generation of perfectly balanced and well-formed rhythms." In Proceedings of the 2016 International Conference on New Interfaces for Musical Expression (NIME 2016). Brisbane, Australia: Griffith University.

Milne, A. J., and S. Rolph. 2014. TiHYL. The Stern Brocot Band. (V. Angelis, S. Holland, A. J. Milne, S. Rolph). SoundCloud, https://soundcloud.com/andrew-j-milne/tihyl-studio-version-2014.

Milne, A. J., W. A. Sethares, and J. Plamondon. 2008. "Tuning continua and keyboard layouts." Journal of Mathematics and Music 2 (1): 1-19.

Miranda, E. R., and J. A. Biles, eds. 2007. Evolutionary Computer Music. London: Springer.

Mongoven, C. P. 2013. "Sonification of multiple Fibonacci-related sequences." Annales Mathematicae et Informaticae 41:175-92.

Morrison, G. 1993. "88 cent equal temperament." Xenharmonikôn 15.

Nelson, G. L. 1996. "Real time transformation of musical material with fractal algorithms." Computers Es Mathematics with Applications 32 (1): 109-16.

Nettheim, N. 1992. "On the spectral analysis of melody." Interface: Journal of New Music Research $21: 135-48$. 
Nierhaus, G. 2009. Algorithmic Composition. Wien/NewYork: Springer.

Papert, S. 1980. Mindstorms: Children, Computers, And Powerful Ideas. New York: Basic Books.

Prechtl, A., A. J. Milne, S. Holland, R. Laney, and D. B. Sharp. 2012. "A MIDI sequencer that widens access to the compositional possibilities of novel tunings." Computer Music Journal 36 (1): 42-54.

Pressing, J. 1988. "Maps as generators of musical design." Computer Music Journal 12 (2): 35-46.

Prusinkiewicz, P. 1986. "Score generation with L-systems." In Proceedings of the 1986 International Computer Music Conference, 455-57.

Prusinkiewicz, P., and A. Lindenmayer. 1990. The Algorithmic Beauty of Plants. New York: SpringerVerlag.

Quinn, I. 2004. "A unified theory of chord quality in equal temperaments." PhD diss., University of Rochester.

Rahn, J. 1986. "Asymmetrical ostinatos in Sub-Saharan music: Time, pitch, and cycles reconsidered." Theory Only: Journal of the Michigan Music Theory Society 9 (7): 23-27.

Rohrmeier, M. 2011. "Towards a generative syntax of tonal harmony." Journal of Mathematics and Music 5 (1): $25-53$.

Soddell, F., and J. Soddell. 2005. "Of Lindenmayer systems, fungi and music." In ACMC05 Generate and Test: Proceedings of the Australasian Computer Music Conference 2005, 143-48.

Unemi, T., and D. Bisig. 2004. "Playing music by conducting BOID agents - A style of interaction in the life with A-Life." In Proceedings of A-Life IX, edited by 546-550.

. 2005. "Music by interaction among two flocking species and human." In Proceedings of the Third International Conference on Generative Systems in Electronic Arts, 171-79. Melbourne, Australia.

Voss, R. F., and J. Clarke. 1978. " $1 / f$ noise' in music: Music from $1 / f$ noise." Journal of the Acoustical Society of America 63 (1): 258-63.

Vuza, D. T. 1992. "Supplementary sets and regular complementary unending canons (part three)." Perspectives of New Music 30 (2): 102-24.

White, G. 1997. "Fibonacci." On Present Tense. Australysis Electroband, (R. Dean, S. Evans, G. White). Tall Poppies TP109, compact disc.

Will, U., and C. Ellis. 1996. "A re-analyzed Australian western desert song: Frequency performance and interval structure." Ethnomusicology 40 (2): 187-222.

Wilson, E. 1975. Letter to Chalmers pertaining to moments-of-symmetry/Tanabe cycle. [PDF document]. Retrieved from http://www.anaphoria.com/mos.pdf.

- 1997. The golden horograms of the scale tree. [PDF document]. Retrieved from http://www. anaphoria.com/hrgm.PDF.

Wishart, T. 1983. On Sonic Art. London: Gordon / Breach.

Xenakis, I. 1971. Formalized Music: Thought and Mathematics in Composition. Bloomington and London: Indiana University Press. 